\title{
UPAR and Cathepsin B Downregulation Induces Apoptosis by Targeting Calcineurin A to BAD via Bcl-2 in Glioma
}

\author{
Rama Rao Malla ${ }^{1}$, Sreelatha Gopinath ${ }^{1}$, Christopher S. Gondi ${ }^{1}$, Kiranmai Alapati ${ }^{1}$, Dzung H. \\ Dinh $^{2}$, Andrew J. Tsung ${ }^{2}$, and Jasti S. Rao ${ }^{1,2,{ }^{*}}$ \\ ${ }^{1}$ Department of Cancer Biology and Pharmacology, University of Illinois College of Medicine at \\ Peoria, One Illini Drive, Peoria, IL 61605, USA \\ ${ }^{2}$ Department of Neurosurgery, University of Illinois College of Medicine at Peoria, One Illini Drive, \\ Peoria, IL 61605, USA
}

\begin{abstract}
Cathepsin B and urokinase plasminogen activator receptor (UPAR) are postulated to play key roles in glioma invasion. Calcineurin is one of the key regulators of mitochondrial-dependent apoptosis, but its mechanism is poorly understood. Hence, we studied subcellular localization of calcineurin after transcriptional downregulation of UPAR and cathepsin B in glioma. In the present study, efficient downregulation of uPAR and cathepsin B increased the translocation of calcineurin A from the mitochondria to the cytosol, decreased $\mathrm{pBAD}$ (S136) expression and its interaction with

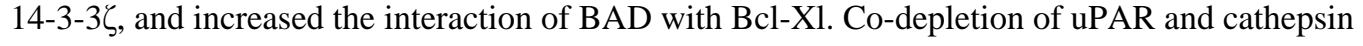
$\mathrm{B}$ induced mitochondrial translocation of BAD and caspase 3 as well as PARP activation, cytochrome $c$ and SMAC release. These effects were inhibited by FK506 $(10 \mu \mathrm{M})$, a specific inhibitor of calcineurin. Calcineurin A was co-localized and also co-immunoprecipitated with $\mathrm{Bcl}-2$. This interaction decreased with co-depletion of UPAR and cathepsin B and also with Bcl-2 inhibitor, HA 14-1 $(20 \mu \mathrm{g} / \mathrm{mL})$. Altered localization and interaction of calcineurin A with Bcl-2 was also observed in vivo when uPAR and cathepsin B were downregulated. In conclusion, downregulation of uPAR and cathepsin $\mathrm{B}$ induced apoptosis by targeting calcineurin A to BAD via Bcl-2 in glioma.
\end{abstract}

\section{Keywords}

Calcineurin; BAD; Bcl2; glioma

\section{INTRODUCTION}

Glioma is a highly complex primary brain disease, which carries a dismal prognosis in spite of aggressive therapy and recent advances in the understanding of the molecular pathways of glioma progression. The unique feature of glioma progression is the deregulation of proteins and genes, such as cathepsin B and uPAR, which play central roles in tumor invasion. Previously, we have demonstrated that both individual or simultaneous downregulation of cathepsin B and uPAR decreased the expression of Bcl-2 and induced apoptosis in glioma cells [1].

Calcineurin [also known as protein phosphatase 3 (formerly protein phosphatase 2B)] is a heterodimer composed of catalytic (Calcineurin A; CnA) and regulatory (Calcineurin B;

\footnotetext{
*Corresponding author: Jasti S. Rao, Ph.D., Professor and Head, Department of Cancer Biology and Pharmacology, University of Illinois College of Medicine, One Illini Drive, Peoria, IL 61605; 309-671-3445 - phone; 309-671-3442 - fax; jsrao@ uic.edu. .
} 
$\mathrm{CnB}$ ) subunits [2]. The $\mathrm{CnB}$ subunit is tightly associated with the $\mathrm{CnA}$ subunit and can only be dissociated under denaturing conditions or at extremely low calcium levels [3]. Within the $\mathrm{CnA}$ subunit, regions with specific assignments have been identified by limited proteolysis [4] and structure determination studies [5]. These include: a catalytic domain, a $\mathrm{CnB}$ binding helix, a calmodulin docking segment, a C-terminal autoinhibitory site, and a Bcl-2 binding domain.

It has been well established that calcineurin is controlled by calcium and calmodulin. In addition, calcineurin is also reported to be regulated by redox mechanisms [6]. Recent studies demonstrated that $\mathrm{Bcl}-2$ regulates calcineurin by sequestration or by shuttling active calcineurin to specific microenvironments and compartments in the cells to interact with other substrates [7]. Upon heterodimerization with the pro-apoptotic protein Bax, Bcl-2 dissociates from calcineurin. Once back in the cytosol, calcineurin starts dephosphorylating BAD [8]. Recently, other calcineurin-mediated functions in the T-cell activation program, muscle growth and development, apoptosis, DNA repair, and cardiac and neural health have been studied [9].

Calcineurin was originally recognized as a key mediator of programmed cell death in $\mathrm{T}$ and B lymphocytes [10,11]. In lymphocytes, calcineurin mediates apoptosis by the induction of Fas and Fas ligand $[12,13]$. In cancer cells, calcineurin induces apoptosis in response to growth factor deprivation as a direct consequence of calcineurin's phosphatase activity [14]. Calcineurin is also reported to mediate ischemia-induced apoptosis via dephosphorylation of Akt [15]. Recently, it has been shown that calcineurin induces apoptosis by dephosphorylation of BAD [8] via cytochrome c/caspase 3-dependent pathway in neuronal cells [16].

In the present study, we first examined the expression levels and localization of calcineurin A in uPAR and cathepsin B-depleted U251 and 4910 glioma cells. We also observed that individual or simultaneous downregulation of UPAR and cathepsin B redistributed calcineurin A and BAD and decreased the expression of pBAD (S112 \& S136). Further, we noticed that FK506, a potent inhibitor of calcineurin, reversed pCU-induced apoptosis and prevented pCU-induced translocation of BAD, cytochrome c, and SMAC. Co-depletion of uPAR and cathepsin B decreased the interaction of calcineurin A with Bcl-2 and increased BAD with Bcl-Xl in both U251 and 4910 glioma cells. Analysis of tissue sections and tissue lysates from pre-established brain tumors (in vivo) further confirmed our in vitro results. Overall, our results show that uPAR and cathepsin B regulate calcineurin via Bcl-2 in glioma.

\section{MATERIALS AND METHODS}

\section{Ethics Statement}

The Institutional Animal Care and Use Committee of the University of Illinois College of Medicine at Peoria (Peoria, IL, USA) approved all surgical interventions and post-operative animal care. The consent was written and approved. The approved protocol number is 851 and is dated November 20, 2009.

\section{Cell lines, chemical reagents and transfection conditions}

U251 glioma cells were obtained from ATCC (American Type Culture Collection, Manassas, VA). 4910 glioma xenograft cells were kindly provided by Dr. David James (University of California-San Francisco). U251 and 4910 cells were grown in DMEM medium and RPMI 1640 medium, respectively and supplemented with 10\% FBS and 1\% penicillin/streptomycin. All primary antibodies used in this study were obtained from Santa Cruz Biotechnology (Santa Cruz, CA) except for anti-BAD, anti-Bcl-2, and anti-Bcl-Xl that 
were obtained from Cell Signaling (Boston, MA) and anti-MTCO2 (Mitochondrial Cytochrome Oxidase subunit 2; a mitochondrial marker) that was obtained from Abcam (Cambridge, MA). Species-specific secondary antibodies conjugated to HRP, Alexa Fluor 488, and Alexa Fluor 595 (Santa Cruz Biotechnology, Santa Cruz, CA) were used in this study. All transfections were carried out using FuGene HD transfection reagent according to the manufacturer's protocol (Roche Applied Science, Madison, WI). Single siRNA constructs directed against $\mathrm{UPAR}(\mathrm{pU})$ and cathepsin $\mathrm{B}(\mathrm{pC})$ and the bicistronic construct directed against both cathepsin B and uPAR (pCU) were used in the present study. Details of siRNA vector construction has been discussed earlier [17]. Cells were transfected for 72 hrs with scrambled vector (pSV), pU, pC, or pCU. For simultaneous overexpression of uPAR and cathepsin B, cells were co-transfected with a plasmid expressing full-length human cDNA clone of uPAR (flU) (SC319092) and cathepsin B (flC) (SC109129). For overexpression of Bcl-2, cells were transfected for $48 \mathrm{hrs}$ with a plasmid expressing fulllength human cDNA clone of Bcl-2 (flBcl-2) (SC125546) (OriGene Technologies, Inc. Rockville, MD). For the pCU and FK506 combination treatment, cells were treated with FK506 $(10 \mu \mathrm{M})($ Sigma, St. Louis, MO) $48 \mathrm{hrs}$ after transfection with pCU. For the flBcl-2 and HA 14-1 combination treatment, cells were treated with HA 14-1 (20 $\mu \mathrm{g} / \mathrm{mL})($ Sigma, St. Louis, MO) $24 \mathrm{hrs}$ after transfection with flBcl-2 and incubated for another $24 \mathrm{hrs}$ before further analysis. Cells were grown to $70-80 \%$ confluence in 2-well chamber slides and then treated with HA $14-1(20 \mu \mathrm{g} / \mathrm{mL})$ for $24 \mathrm{hrs}$.

\section{Subcellular fractionation, Western blotting, and immunoprecipitation}

Fractionation of mitochondrial and cytosolic proteins was carried out as described previously [1]. For Western blot analysis, equal amounts of protein fractionated on SDSPAGE were immunoblotted with primary antibodies and subsequently incubated with species-specific, HRP-conjugated secondary antibodies (Santa Cruz Biotechnology, CA). Signals were detected using the ECL Western blotting detection system (Pierce, Rockford, IL). Immunoprecipitation assays were carried out by incubating a minimum of $800 \mu \mathrm{g}$ total cell lysate (or cytosolic or mitochondrial fractions) with antibody for $3 \mathrm{hrs}$ at room temperature or overnight at $4^{\circ} \mathrm{C}$ on a rotating shaker. Protein $\mathrm{A} / \mathrm{G}$ agarose beads (Miltenyi Biotec, Auburn, CA) were added to the above complex and incubated for either $30 \mathrm{~min}$ on ice or overnight at $4^{\circ} \mathrm{C}$. These beads were passed through $20 \mu \mathrm{M}$ columns following the manufacturer's instructions and immunoprecipitates were immunoblotted with primary antibodies.

\section{Determination of mitochondrial membrane potential}

Mitochondrial membrane potential changes were assayed with MitoLight dye according to the manufacturer's instructions (Millipore, Danvers, MA). U251 and 4910 cells were transfected with pSV and pCU in the presence or absence of FK506 $(10 \mu \mathrm{M})$. Cells were then incubated for $30 \mathrm{~min}$ with pre-diluted MitoLight solution. Cells were washed twice with $1 \mathrm{X}$ incubation buffer and FACS analysis was carried out.

\section{APO-BrdU TUNEL assay}

Apoptotic cells were detected using the APO-BrdU TUNEL kit (Molecular Probes Inc, Eugene, OR) according to the manufacturer's protocol for flow cytometry. Briefly, U251 and 4910 cells were harvested $72 \mathrm{hrs}$ after transfection in the presence or absence of FK506 and fixed with $4 \%$ paraformaldehyde for $30 \mathrm{~min}$ at room temperature. Cells were washed twice in PBS, re-suspended in cold ethanol, and then incubated with $50 \mu \mathrm{L}$ DNA-labeling solution containing TdT enzyme and BrdUTP at $37^{\circ} \mathrm{C}$ for $60 \mathrm{~min}$. Cells were washed with PBS and stained with Alexa Fluor 488 dye-labeled anti-BrdU antibody for $30 \mathrm{~min}$. APOBrdU-TUNEL positive cells were measured by emission of Alexa Fluor 488 intensity 
through flow cytometry analysis. For negative control, TdT enzyme was replaced with the reaction buffer in the DNA-labeling solution.

\section{Annexin V/PE staining assay}

Cells transfected with pCU in the presence or absence of FK506 were harvested, washed and re-suspended in $500 \mu \mathrm{L}$ of binding buffer. Then, cells were stained with $5 \mu \mathrm{L}$ of Annexin VPE (BioVision, CA) according to the manufacturer's instructions. Cells positive for Annexin V-PE were analyzed by flow cytometry $(E X=488 ; E m=578)$ using the phycoerythrin emission signal detector. Data acquisition and analysis were performed using a Becton Dickinson flow cytometer with Cell Quest software.

\section{Immunofluoresence}

For immunofluorescence assay, cells grown in 2-well chamber slides were washed with PBS, fixed, permeabilized with ice-cold methanol and blocked with normal goat serum (Invitrogen, Carlsbad, CA). Cells were incubated overnight with primary antibodies at $4{ }^{\circ} \mathrm{C}$, washed with PBS, and incubated with fluorescent-labeled, species-specific secondary antibodies (Alexa Fluor ${ }^{\circledR}$ ) for $1 \mathrm{hr}$ at room temperature. Before mounting, cells were incubated for a brief period with DAPI for nuclear staining and analyzed with a confocal microscope (Olympus BX61 Fluoview, Minneapolis, MN). Overlay of images was done using SPOT Advanced software (Windows version 4.0.8).

\section{Immunohistochemical analysis of brain tumor sections}

Stereotactic implantation of U251 and 4910 cells, followed by treatment with mock (PBS), pSV and pCU using Alzet minipumps at flow rate of $0.25 \mu \mathrm{L} / \mathrm{hr}$ was carried out as previously described $[18,19]$. Once control animals showed chronic symptoms (approximately 30 days), the animals were euthanized by cardiac perfusion using $10 \%$ buffered formalin. The brains were removed, stored in $10 \%$ formalin, and embedded in paraffin following standard protocols. Immunoblot analysis was carried out on fresh brain tissues. Paraffin-embedded tumors sections were subjected to rehydration by passing through a series of xylene and $100 \%$ and $90 \%$ ethanol as described previously $[1,20]$. Tumor sections were double stained for calcineurin A and Bcl-2 and analyzed as described earlier.

\section{RESULTS}

\section{Downregulation of UPAR and cathepsin B redistributed calcineurin A}

Previously, we have demonstrated that knockdown of uPAR and cathepsin B using siRNA significantly decreased the expression of Bcl-2 and induced apoptosis in glioma cells [1]. The present study was carried out to further elucidate the underlying molecular mechanism of apoptosis. Western blotting showed that UPAR and cathepsin B expression was efficiently decreased with $\mathrm{pU}, \mathrm{pC}$ and $\mathrm{pCU}$ (Fig. 1A). Quantification of protein bands revealed that uPAR expression decreased $80 \%$ in U251 cells and $70 \%$ in 4910 cells with $\mathrm{pU}(\mathrm{p}<0.001)$. Similarly, cathepsin B expression decreased $70 \%$ in U251 cells and $80 \%$ in 4910 cells with $\mathrm{pC}(\mathrm{p}<0.001)$. Interestingly, expression of uPAR and cathepsin B decreased $90 \%$ and $95 \%$, respectively in pCU-transfected $\mathrm{U} 251$ and 4910 cells $(\mathrm{p}<0.001)$.

Calcineurin was previously reported in different cellular compartments [21]. As a preliminary study, immunocytochemical analysis of calcineurin A and mitochondrial cytochrome oxidase subunit-2 (MTCO2) was carried out on the monolayers of U251 and 4910 cells. Co-localization of calcineurin A and MTCO2 confirmed the presence of calcineurin A in mitochondria (Supplementary Fig. S1A). To evaluate the impact of siRNAmediated downregulation of UPAR and cathepsin B on calcineurin-mediated apoptosis in 
U251 and 4910 cells, immunoblot analysis was performed on total lysates using antibodies specific for calcineurin A and B. Individual or simultaneous knockdown of uPAR and cathepsin B did not alter the expression of either calcineurin A and B in total lysates (data not shown). Cell fractionation (mitochondrial versus cytoplasmic) was utilized in conjugation with Western blotting to determine changes in calcineurin A localization after transient transfection with pU, pC and pCU in U251 and 4910 cells. Transfection with pU, $\mathrm{pC}$ and $\mathrm{pCU}$ significantly reduced mitochondrial membrane-associated calcineurin $\mathrm{A}$ and increased cytoplasmic levels of calcineurin A in both U251 and 4910 cells (Fig. 1B). Densitometric analysis of protein bands revealed that calcineurin A levels increased $\sim 1.8$ fold with both $\mathrm{pU}$ and $\mathrm{pC}$ and $\sim 2.4$ fold with $\mathrm{pCU}$ ( $\mathrm{p}<0.001$ ) in cytoplasmic fractions of both U251 and 4910 cells. However, its levels were significantly decreased with pU, pC and pCU (p<0.001) in mitochondrial fractions of both U251 and 4910 cells. Although, calcineurin $\mathrm{B}$ was detected on the mitochondrial membrane, its levels were not altered with $\mathrm{pU}, \mathrm{pC}$ and $\mathrm{pCU}$ transfection (data not shown).

\section{Effect of UPAR and cathepsin B downregualtion on expression of BAD and PBAD}

It has been previously reported that calcineurin regulates BAD-mediated apoptosis in neuronal cells by dephosphoryation of BAD [8]. Hence, we checked the potential changes in BAD levels after transfection with pU, pC and pCU in U251 and 4910 cells. The results indicated no significant change in the expression of BAD in total cell lystes after treatments as compared to the control (Fig. 2A).

When probed for pBAD S112, pBAD S136 and pBAD S155, a significant reduction was observed in the expression of pBAD S136 and a moderate decrease was noticed in pBAD S112 with downregulation of uPAR and cathepsin B in both U251 and 4910 cells. The expression of pBAD S155 was unaffected with the treatment in both cell lines (Fig. 2A).

Immunoblot analysis of the cytosol fractions showed that the amounts of BAD were significantly decreased after transfection with $\mathrm{pU}, \mathrm{pC}$ and $\mathrm{pCU}$ as compared to controls in both $\mathrm{U} 251$ and 4910 cells. We next examined the amount of BAD in the mitochondrial fractions and it was noted that the amount of mitochondrial BAD significantly increased after treatment with pU, pC and pCU in both $\mathrm{U} 251$ and 4910 cell lyastes as compared to controls (Fig. 2B). To further confirm the effect of uPAR and cathepsin B downregulation on the expression of BAD, U251 and 4910 cells were transfected with full-length uPAR (flU) and cathepsin B (flC) expressing plasmids. Western blotting analysis showed that, in both U251 and 4910 cells, simultaneous overexpression of uPAR and cathepsin B increased BAD levels in cytosolic fractions while decreasing BAD levels in mitochondrial fractions, thereby confirming earlier results (Supplementary Fig. S1B).

Calcineurin-mediated dephosphorylation of BAD will result in its dissociation from the 14-3-3-BAD complex and subsequent binding to Bcl-Xl [8]. As the levels of phosphorylated BAD (S136) decreased significantly with pCU treatment, we predicted that dissociation of BAD from 14-3-3-BAD complex would also occur with the same treatment. Immunofluorescence staining was used to examine co-localization of BAD with 14-3-3 and Bcl-xl in U251 and 4910 cells after pSV and pCU treatments. In pSV-treated U251 and 4910 cells, yellow staining on confocal images (overlay) indicates co-localization of pBAD (S136) with 14-3-3 $\zeta$ in contrast to pCU-treated cells (Supplemental Fig. S2A). However, in pCU-transfected U251 and 4910 cells, yellow staining on overlay of BAD and Bcl-xl images shows co-localization between BAD and Bcl-xl in contrast to controls (Supplemental Fig. S2B). To provide additional evidence, immunoprecipitation experiments were conducted on cytosolic and mitochondrial fractions using an antibody specific for BAD. We found that the amount of 14-3-3 $\zeta$ in BAD immunoprecipitates significantly decreased in cytosolic fractions of both $\mathrm{U} 251$ and 4910 cells with pU, pC and pCU treatments as 
compared to controls (Fig. 2C). In contrast, the amount of Bcl-Xl dramatically increased in mitochondrial fractions of cells treated with $\mathrm{pU}, \mathrm{pC}$ and $\mathrm{pCU}$, indicating rapid dissociation of BAD from 14-3-3 in cytosolic fractions and association with Bcl-xl in mitochondrial fractions (Fig. 2C)

\section{Effect of calcineurin inhibitor, FK506, on pCU-induced apoptosis}

Previously, we have demonstrated that transient transfection of glioma cells with pCU efficiently induced apoptosis [1]. To investigate calcineurin-mediated regulation of apoptosis in U251 and 4910 glioma cells, we performed flow cytometry analysis with APOBrdU-TUNEL after transfection with pCU in the presence or absence of FK506 $(10 \mu \mathrm{M})$. The percentage of glioma cells with nuclear DNA strand breaks (TUNEL-positive cells) was calculated on the basis of results obtained from the histogram analysis (Fig. 3A). The proportion of TUNEL-positive cells was significantly higher in pCU-treated U251 cells $(49.22 \%)$ as compared to untreated controls $(7.45 \%)$ or SV $(6.79 \%)$. Similarly, the proportion of TUNEL-positive cells was $52.5 \%, 13.7 \%$ and $13.4 \%$ in pCU-treated, pSVtreated, and untreated 4910 cells, respectively. However, FK506 reduced pCU-induced TUNEL-positive cells in U251 (12.49\%) and 4910 cells (19.84\%), thereby indicating that pCU-induced apoptosis might be regulated by calcineurin.

Flow cytometry with Annexin V-PE staining was carried out to further confirm calcineurinmediated apoptosis in pCU-treated glioma cells. The results obtained from the histogram analysis indicate that the percentage of Annexin V-PE-positive cells were significantly high in pCU-treated U251 cells (64.68\%) as compared to pSV-treated (4.92) or untreated (4.25\%) controls (Fig. 3B). In 4910 cells, the Annexin V-PE-positive cells were $42.09 \%$ with pCU treatment as compared to pSV-treated (5.45\%) or untreated (5.22\%) controls (Fig. 3B). However, Annexin V-PE-positive cells were reduced significantly with FK506 in pCUtransfected U251 (10\%) and 4910 (15.89\%) cells, which further confirms calcineurinmediated apoptosis in uPAR and cathepsin B-depleted glioma cells.

PARP cleavage occurs early as a result of the activity of caspase 3 and is associated with the condensed chromatin in apoptotic cells, which is a measure of apoptosis. Activation of caspase 3 and PARP was analyzed in pCU-treated U251 and 4910 cells in the presence or absence of FK506 $(10 \mu \mathrm{M})$ using western blot analysis. The results indicate increased levels of cleaved caspase 3 and PARP in pCU-transfected U251 and 4910 cells; however levels decreased to basal levels after FK506 treatment in pCU-transfected cells (Fig. 4A).

Previously, we have demonstrated that pCU rapidly decreased mitochondrial membrane potential in glioma cells [1]. To evaluate the role of calcineurin in mitochondrial-mediated apoptosis, U251 and 4910 cells were treated with pCU in the presence or absence of FK506, and changes in mitochondrial membrane potential $(\Delta \Psi \mathrm{m})$ were determined using flow cytometry with MitoLight dye. The histogram analysis revealed high mitochondrial membrane potential (80\%) in both U251 and 4910 control cells as compared to pCU-treated U251 (31\%) and 4910 (34\%) cells (Supplemental Fig. S2C). However, FK506 restored membrane potential to $78 \%$ in pCU-treated U251 and 4910 cells.

To further confirm, we analyzed levels of BAD, cytochrome c and SMAC in cytosolic and mitochondrial fractions that were obtained from pCU-transfected U251 and 4910 cells in the presence or absence of FK506. Western blotting results showed that FK506 treatment increased BAD levels in cytosolic fractions but decreased them in mitochondrial fractions to basal levels in pCU-transfected U251 and 4910 cells. However, pro-apoptotic molecules cytochrome $\mathrm{c}$ and SMAC levels decreased in cytosolic fractions but increased in mitochondrial fractions of pCU-transfected U251 and 4910 cells in presence of FK506 (Fig. 
4B). These results confirm calcineurin mediated regulation of BAD and its downstream apoptotic mediators, cytochrome $\mathrm{c}$ and SMAC, in glioma cells.

\section{Effect of UPAR and cathepsin B downregulation on interaction of calcineurin A with Bcl-2}

It has been reported that Bcl-2, an anti-apoptotic protein, inhibits calcineurin activity through physical interaction [21]. As downregulation of uPAR and cathepsin B decreased the expression of Bcl-2 in both U251 and 4910 cells (Supplementary Fig. S3A), we hypothesized that the same treatment would decrease the interaction of Bcl-2 with calcineurin A. To study the interaction between calcineurin A and Bcl-2, we carried out double immunostaining on monolayers of pCU-transfected U251 and 4910 cells using antibodies specific for calcineurin A and Bcl-2. Merged figures showed strong colocalization (yellow color) of calcineurin A with Bcl-2 in controls cells (Fig. 5A). As expected, pCU treatment significantly reduced the co-localization of calcineurin A and Bcl-2 in U251 and 4910 cells. Immunoblot analysis (Fig 1A) and immunocyto chemistry revealed that calcineurin translocation from mitochondira to cytoplasm and decreased expression of Bcl-2 with pCU treatments resulted in less interaction of Bcl-2 and calcineurin. The distribution of calcineurin indicated the translocation of calcineurin to cytoplasm from mitochondria in pCU-treated cells compared to controls further confirming the results obtained in Fig 1A. Similarly, HA 14-1 a functional inhibitor of Bcl-2, also showed decreased yellow staining in both U251 and 4910 cells (Fig. 5B).

To validate the results obtained with the double-labeling experiments, we studied the interaction between $\mathrm{Bcl}-2$ and calcineurin A using immunoprecipitation of $\mathrm{Bcl}-2$ with specific antibody in untreated, pSV-transfected, and pCU-transfected U251 and 4910 cell lysates. High calcineurin A and Bcl-2 levels were detected by Western blotting in anti-Bcl-2 immunoprecipitates of untreated or pSV-treated U251 and 4910 cells, which significantly decreased with pCU treatment (Fig. 5C). In contrast, as compared to untreated controls, Bcl-2 and calcineurin A levels were significantly higher in both U251 and 4910 cells, in which uPAR and cathepsin B were overexpressed by double transfection with full-length uPAR and cathepsin B cDNA plasmids (Supplemental Fig. 3B). To further confirm the interaction between Bcl-2 and calcineurin A, Bcl-2 was overexpressed in both $\mathrm{U} 251$ and 4910 cells using full-length Bcl-2 cDNA plasmid. Expression of Bcl-2 was analyzed by Western blotting. The results show that Bcl-2 was overexpressed in U251 and 4910 glioma cells with flBcl-2 treatment (Fig. 5D). Next, Bcl-2 was immunoprecipitated in Bcl-2overexpressed cells. Western blotting of Bcl-2 immunoprecipitates revealed that Bcl-2 and calcineurin A levels were significantly higher in Bcl-2-overexpressed cells as compared to controls (Fig. 5E). However, the use of a Bcl-2 functional inhibitor (HA 14-1) significantly decreased calcineurin A levels in Bcl-2 immunoprecipitates of Bcl-2-overexpressed U251 and 4910 cells; however, there was no change in Bcl-2 levels (Fig. 5F). These results confirm that uPAR and cathepsin B regulate calcineurin A via Bcl-2.

\section{pCU decreases interaction of calcineurin A with $\mathrm{Bcl}-2$ in pre-established intracranial tumors}

From our in vitro studies, we inferred that knockdown of uPAR and cathepsin B induced apoptosis by altering localization of calcineurin A and BAD via Bcl-2. Thus, we next decided to study the effect of pCU on pre-established gliomas in nude mice. To evaluate the mechanism by which apoptosis is induced in nude mice brains with tumors, expression levels of uPAR, cathepsin B, calcineurin A and BAD were evaluated in U251 and 4910 brain tissue lysates using Western blotting analysis. The results show that uPAR and cathepsin B levels were significantly reduced but there was no change in calcineurin A and BAD levels in pCU-treated brain whole tissue lysates as compared to controls (Fig. 6A). Furthermore, cytosolic and mitochondrial fractions of tissue lysates were evaluated by 
immunoblot analysis. We observed that calcineurin A levels were significantly higher but BAD levels were lower in cytosolic fractions of U251 and 4910 tissue lysates. In contrast, calcineurin A levels were significantly lower but BAD levels were higher in mitochondrial fractions. Further, we checked whether Bcl-2, involved in the regulation of calcineurin A in the mice tumor brains, was similar to in vitro results. The interaction between $\mathrm{Bcl}-2$ and calcineurin A was studied by immunoprecipitation of Bcl-2 with specific antibody in untreated, pSV-treated and pCU-treated U251 and 4910 tissue lysates. Significantly increased amounts of calcineurin A bound to Bcl-2 were observed in anti-Bcl-2 immunoprecipitates of untreated or $\mathrm{pSV}$-treated tissue lysates; however, a significant reduction was noticed in pCU-treated lysates (Fig. 6B). To further confirm, we carried out immunostaining of $\mathrm{U} 251$ and 4910 brain tissue sections using calcineurin A and Bcl-2 antibodies. As expected, calcineurin A had a strong interaction with Bcl-2 in controls (yellow stain) but this was significantly decreased with pCU treatment (Fig. 6C). These results indicate that in tumor cells there is a strong interaction between calcineurin A (Green in U87 and Red in 4910) and Bcl-2 (Red in U87 and Green in 4910). The pCU-treated tumor sections showed significant reduction in the interaction as seen by the reduced yellow color (co-localization of red and green).

\section{DISCUSSION}

In the progression of cancer, cells acquire a variety of capabilities that promote tumor growth and invasion. Acquisition of these capabilities involves signaling interactions associated with the cathepsin B and uPAR system [22]. Several studies have demonstrated that uPAR and cathepsin B are overexpressed in high-grade glioma, and this overexpression correlates strongly with invasive phenotype and poor prognosis [23, 24]. Cathepsin B has been shown to initiate a proteolytic cascade involving uPAR. Hence, this system has been identified as a potential therapeutic target. RNA interference (RNAi) has emerged as a powerful tool in the exploration of target genes. In our previous report, we observed that transfection of glioma cells with siRNA for uPAR and cathepsin B effectively induced apoptosis [1].

Modulation of the phosphorylation state of proteins regulated by phosphatases has emerged as an important mechanism of apoptotic regulation. In contrast to the multitude of protein kinases, relatively few protein phosphatases are known and their regulation is poorly understood. Among the phosphatases, calcineurin was previously shown to be involved in the regulation of apoptosis [8]. Moreover, calcineurin has been implicated in both transcription-dependent and -independent apoptosis. It was reported that calcineurin induced transcription-independent apoptosis by dephosphorylating BAD [8].

The present study was carried out to explore the role of calcineurin in apoptosis induced by downregulation of uPAR and cathepsin B in human glioma. We noticed that uPAR and cathepsin B downregulation redistributed calcineurin A from the mitochondria to cytosol in U251 and 4910 cells. Shibasaki, et al. [21] reported that Bcl-2 interacts strongly with the catalytic subunit $(\mathrm{CnA})$ when in complex with the regulatory subunit $(\mathrm{CnB})$. Moreover, Reed [25] reported that Bcl-2 overexpression causes the redistribution of calcineurin from the cytosol to the mitochondrial membrane and prevents interaction with calcineurin substrates in the cytosol.

The Bcl-2 family of proteins is important to the regulation of cell death in cancer cells. Apoptosis is determined by the relative balance between pro-apoptotic molecules and antiapoptotic molecules [26-28]. Depending upon its phosphorylation status, the pro-apoptotic molecule BAD promotes both cancer cell survival and apoptosis. The present study demonstrated that both individual and simultaneous downregulation of uPAR and cathepsin 
B dephosphorylated BAD at S112 and S136 in both U251 and 4910 cells. Interestingly, Masters, et al. reported that upon phosphorylation at both S112 and S136, BAD binds to 14-3-3 proteins; however, pS136 may be the more predominant 14-3-3 binding site [29]. BAD dephosphorylation may not alter its association with 14-3-3 proteins, but it may further influence its subcellular localization. In this study, we observed that downregulation of uPAR and cathepsin B caused translocation of BAD from cytosol to mitochondria in both U251 and 4910 glioma cells and vice versa when uPAR and cathepsin B were overexpressed. In addition, we observed that the interaction of BAD decreased with 14-3-3 $\zeta$ but increased with Bcl-xl in uPAR and cathepsin B-depleted U251 and 4910 cells.

Previously, Zha, et al. reported that phosphorylation-dependent association of BAD with 14-3-3 proteins shifts its localization from mitochondria to the cytosol [28].

Dephosphorylated BAD promotes cell death at least in part through heterodimerization with the anti-apoptotic protein Bcl-xl [30]. Although S136 is the apical serine, the phosphorylation of which is needed for neutralizing BAD's apoptotic function, pS112 dephosphorylation may be the initial dephosphorylation event required for promoting the anti-apoptotic activity of BAD [31].

Several BAD phosphatases have been characterized within specific death paradigms, including calcineurin [32]. In the present study, we found that FK506, a specific inhibitor of calcineurin, rescued glioma cells from pCU-induced apoptosis and protected cells from loss of mitochondrial membrane potential, thereby indicating the role of calcineurin in mitochondrial-mediated apoptosis. In addition, FK506 significantly inhibited pCU-induced translocation of BAD from the cytosol to mitochondria in U251 and 4910 cells. FK506 inhibited the release of cytochrome $\mathrm{c}$ and SMAC from the mitochondria to cytosol in pCUtransfected U251 and 4910 cells. Further, FK506 also inhibited pCU-activated caspase 3 and PARP in U251 and 4910 glioma cells. However, Hortelano, et al. [33] reported that FK506 protected macrophages from nitric oxide-dependent apoptosis. Yardin, et al. [34] reported that FK506 blocked neuronal apoptosis induced by serum deprivation in rat neuronal cultures. Recently, [35] reported that FK506 increased apoptotic cell death in a caspasedependent manner in PC12 cells.

Current evidence suggests that some phosphatases do not find their physiological substrates by simple diffusion, and they are frequently directed to their substrates by interaction with other proteins [36]. The proportion of phosphatase directed to BAD is determined by the amount of targeting protein synthesized [37]. It was reported that calcineurin bound to Bcl-2 in mitochondria suppresses NF-AT pathway [21] or BAD dephosphorylation [8]. Previously, we reported that uPAR and cathepsin B downregulation decreased Bcl-2 and increased BAX in U251 and 5310 cells [1]. In line with these results and confirmed by immunofluoresence and immunoprecipitation assays, we observed a low level of Bcl-2/ calcineurin A association in pCU-treated U251 and 4910 cells; Bcl-2 was the limiting molecule in the formation of this complex. However, overexpression of uPAR and cathepsin B (or Bcl-2) enhanced interaction of Bcl-2 with calcineurin A. However, HA 14-1 (a Bcl-2 inhibitor) decreased this association in Bcl-2-overexpressed U251 and 4910 cells. Earlier, it was reported that BAX interferes in the interaction of Bcl-2 with calcineurin, possibly by displacing calcineurin from Bcl-2 [38]. These results suggest that Bcl-2 has either a direct or an indirect role in controlling calcineurin distribution.

It has been previously demonstrated that in vivo treatment of pre-established intracranial tumors with plasmids expressing siRNA for uPAR and cathepsin B significantly inhibited tumor growth in glioma $[1,39]$. In our study, we observed that apoptosis in pCU-treated brain tumors was caused by the redistribution of calcineurin A and BAD. Our colocalization experiments in brain tissue sections and co-immunoprecipitation experiments of pCU-treated brain tissue lysate fractions confirmed decreased association of Bcl-2 and 
calcineurin A in vivo. In conclusion, the redistribution of calcineurin, the reduced association of calcineurin A and Bcl-2, and the dephosphorylation of BAD S136 all play important roles in inducing apoptosis when UPAR and cathepsin B are downregulated in glioma.

\section{Supplementary Material}

Refer to Web version on PubMed Central for supplementary material.

\section{Acknowledgments}

Funding: This research was supported by National Institutes of Health, CA116708 (to JSR). Contents of this manuscript are solely the responsibility of the authors and do not necessarily represent the official views of NIH.

\section{Reference List}

1. Malla R, Gopinath S, Alapati K, Gondi CS, Gujrati M, Dinh DH, Mohanam S, Rao JS. Downregulation of uPAR and cathepsin B induces apoptosis via regulation of Bcl-2 and Bax and inhibition of the PI3K/Akt pathway in gliomas. PLoS One. 2010; 5:e13731. [PubMed: 21060833]

2. Klee CB, Draetta GF, Hubbard MJ. Calcineurin. Adv Enzymol Relat Areas Mol Biol. 1988; 61:149-200. 149-200. [PubMed: 2833077]

3. Stemmer PM, Klee CB. Dual calcium ion regulation of calcineurin by calmodulin and calcineurin B. Biochemistry. 1994; 33:6859-6866. [PubMed: 8204620]

4. Hubbard MJ, Klee CB. Functional domain structure of calcineurin A: mapping by limited proteolysis. Biochemistry. 1989; 28:1868-1874. [PubMed: 2541767]

5. Kissinger CR, Parge HE, Knighton DR, Lewis CT, Pelletier LA, Tempczyk A, Kalish VJ, Tucker KD, Showalter RE, Moomaw EW. Crystal structures of human calcineurin and the human FKBP12FK506-calcineurin complex. Nature. 1995; 378:641-644. [PubMed: 8524402]

6. Wang X, Culotta VC, Klee CB. Superoxide dismutase protects calcineurin from inactivation. Nature. 1996; 383:434-437. [PubMed: 8837775]

7. Erin N, Bronson SK, Billingsley ML. Calcium-dependent interaction of calcineurin with Bcl-2 in neuronal tissue. Neuroscience. 2003; 117:541-555. [PubMed: 12617961]

8. Wang HG, Pathan N, Ethell IM, Krajewski S, Yamaguchi Y, Shibasaki F, McKeon F, Bobo T, Franke TF, Reed JC. Ca2+-induced apoptosis through calcineurin dephosphorylation of BAD. Science. 1999; 284:339-343. [PubMed: 10195903]

9. Martin GK, Ohlms LA, Franklin DJ, Harris FP, Lonsbury-Martin BL. Distortion product emissions in humans. III. Influence of sensorineural hearing loss. Ann Otol Rhinol Laryngol Suppl. 1990; 147:30-42. [PubMed: 2110798]

10. Fruman DA, Mather PE, Burakoff SJ, Bierer BE. Correlation of calcineurin phosphatase activity and programmed cell death in murine T cell hybridomas. Eur J Immunol. 1992; 22:2513-2517. [PubMed: 1382988]

11. Zhao Y, Tozawa Y, Iseki R, Mukai M, Iwata M. Calcineurin activation protects T cells from glucocorticoid-induced apoptosis. J Immunol. 1995; 154:6346-6354. [PubMed: 7539018]

12. Lobo FM, Zanjani R, Ho N, Chatila TA, Fuleihan RL. Calcium-dependent activation of TNF family gene expression by $\mathrm{Ca} 2+/$ calmodulin kinase type IV/Gr and calcineurin. J Immunol. 1999; 162:2057-2063. [PubMed: 9973478]

13. Toth R, Szegezdi E, Molnar G, Lord JM, Fesus L, Szondy Z. Regulation of cell surface expression of Fas (CD95) ligand and susceptibility to Fas (CD95)-mediated apoptosis in activation-induced T cell death involves calcineurin and protein kinase C, respectively. Eur J Immunol. 1999; 29:383393. [PubMed: 10064053]

14. Shibasaki F, McKeon F. Calcineurin functions in $\mathrm{Ca}(2+)$-activated cell death in mammalian cells. J Cell Biol. 1995; 131:735-743. [PubMed: 7593193] 
15. Park CH, Kim YS, Kim YH, Choi MY, Yoo JM, Kang SS, Choi WS, Cho GJ. Calcineurin mediates AKT dephosphorylation in the ischemic rat retina. Brain Res. 2008; 1234:148-157. [PubMed: 18703031]

16. Asai A, Qiu J, Narita Y, Chi S, Saito N, Shinoura N, Hamada H, Kuchino Y, Kirino T. High level calcineurin activity predisposes neuronal cells to apoptosis. J Biol Chem. 1999; 274:34450-34458. [PubMed: 10567426]

17. Gondi CS, Kandhukuri N, Kondraganti S, Gujrati M, Olivero WC, Dinh DH, Rao JS. RNA interference-mediated simultaneous down-regulation of urokinase-type plasminogen activator receptor and cathepsin B induces caspase-8-mediated apoptosis in SNB19 human glioma cells. Mol Cancer Ther. 2006; 5:3197-3208. [PubMed: 17172424]

18. Gondi CS, Lakka SS, Dinh DH, Olivero WC, Gujrati M, Rao JS. Intraperitoneal injection of an hpRNA-expressing plasmid targeting uPAR and uPA retards angiogenesis and inhibits intracranial tumor growth in nude mice. Clin Cancer Res. 2007; 13:4051-4060. [PubMed: 17634529]

19. Lakka SS, Gondi CS, Yanamandra N, Olivero WC, Dinh DH, Gujrati M, Rao JS. Inhibition of cathepsin B and MMP-9 gene expression in glioblastoma cell line via RNA interference reduces tumor cell invasion, tumor growth and angiogenesis. Oncogene. 2004; 23:4681-4689. [PubMed: 15122332]

20. Gopinath S, Malla RR, Gondi CS, Alapati K, Fassett D, Klopfenstein JD, Dinh DH, Gujrati M, Rao JS. Co-depletion of cathepsin B and uPAR induces G0/G1 arrest in glioma via FOXO3a mediated p27 upregulation. PLoS One. 2010; 5:e11668. [PubMed: 20661471]

21. Shibasaki F, Kondo E, Akagi T, McKeon F. Suppression of signalling through transcription factor NF-AT by interactions between calcineurin and Bcl-2. Nature. 1997; 386:728-731. [PubMed: 9109491]

22. Aguirre-Ghiso JA, Alonso DF, Farias EF, Gomez DE, Kier Joffe EB. Deregulation of the signaling pathways controlling urokinase production. Its relationship with the invasive phenotype. Eur J Biochem. 1999; 263:295-304. [PubMed: 10406935]

23. Mohanam S, Sawaya R, McCutcheon I, Ali-Osman F, Boyd D, Rao JS. Modulation of in vitro invasion of human glioblastoma cells by urokinase-type plasminogen activator receptor antibody. Cancer Res. 1993; 53:4143-4147. [PubMed: 8395977]

24. Sivaparvathi M, Sawaya R, Wang SW, Rayford A, Yamamoto M, Liotta LA, Nicolson GL, Rao JS. Overexpression and localization of cathepsin B during the progression of human gliomas. Clin Exp Metastasis. 1995; 13:49-56. [PubMed: 7820956]

25. Reed JC. Double identity for proteins of the Bcl-2 family. Nature. 1997; 387:773-776. [PubMed: 9194558]

26. del Peso L, Gonzalez-Garcia M, Page C, Herrera R, Nunez G. Interleukin-3-induced phosphorylation of BAD through the protein kinase Akt. Science. 1997; 278:687-689. [PubMed: 9381178]

27. Scheid MP, Duronio V. Dissociation of cytokine-induced phosphorylation of Bad and activation of PKB/akt: involvement of MEK upstream of Bad phosphorylation. Proc Natl Acad Sci U S A. 1998; 95:7439-7444. [PubMed: 9636168]

28. Zha J, Harada H, Yang E, Jockel J, Korsmeyer SJ. Serine phosphorylation of death agonist BAD in response to survival factor results in binding to 14-3-3 not BCL-X(L). Cell. 1996; 87:619-628. [PubMed: 8929531]

29. Masters SC, Yang H, Datta SR, Greenberg ME, Fu H. 14-3-3 inhibits Bad-induced cell death through interaction with serine-136. Mol Pharmacol. 2001; 60:1325-1331. [PubMed: 11723239]

30. Danial NN. BAD: undertaker by night, candyman by day. Oncogene. 2008; 27(Suppl 1):S53-S70. [PubMed: 19641507]

31. Chiang CW, Kanies C, Kim KW, Fang WB, Parkhurst C, Xie M, Henry T, Yang E. Protein phosphatase $2 \mathrm{~A}$ dephosphorylation of phosphoserine 112 plays the gatekeeper role for BADmediated apoptosis. Mol Cell Biol. 2003; 23:6350-6362. [PubMed: 12944463]

32. Jacobsson S, Jonsson L, Rank F, Rothman U. Studies on healing of Debrisan-treated wounds. Scand J Plast Reconstr Surg. 1976; 10:97-101. [PubMed: 1019590] 
33. Hortelano S, Lopez-Collazo E, Bosca L. Protective effect of cyclosporin A and FK506 from nitric oxide-dependent apoptosis in activated macrophages. Br J Pharmacol. 1999; 126:1139-1146. [PubMed: 10205001]

34. Yardin C, Terro F, Lesort M, Esclaire F, Hugon J. FK506 antagonizes apoptosis and c-jun protein expression in neuronal cultures. Neuroreport. 1998; 9:2077-2080. [PubMed: 9674596]

35. Takadera T, Sakamoto Y, Hizume Y, Ohyashiki T. Cyclosporine A- and FK506-induced apoptosis in PC12 cells. Cell Biol Toxicol. 2007; 23:355-360. [PubMed: 17385075]

36. Colledge M, Scott JD. AKAPs: from structure to function. Trends Cell Biol. 1999; 9:216-221. [PubMed: 10354567]

37. Ayllon V, Cayla X, Garcia A, Roncal F, Fernandez R, Albar JP, Martinez C, Rebollo A. Bcl-2 targets protein phosphatase 1 alpha to Bad. J Immunol. 2001; 166:7345-7352. [PubMed: 11390485]

38. Rong YP, Barr P, Yee VC, Distelhorst CW. Targeting Bcl-2 based on the interaction of its BH4 domain with the inositol 1,4,5-trisphosphate receptor. Biochim Biophys Acta. 2009; 1793:971978. [PubMed: 19056433]

39. Gondi CS, Lakka SS, Dinh D, Olivero W, Gujrati M, Rao JS. Downregulation of uPA, uPAR and MMP-9 using small, interfering, hairpin RNA (siRNA) inhibits glioma cell invasion, angiogenesis and tumor growth. Neuron Glia Biology. 2004; 1:165-176. [PubMed: 16804563] 
A)

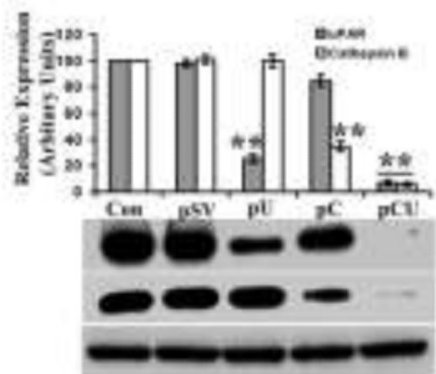

i)

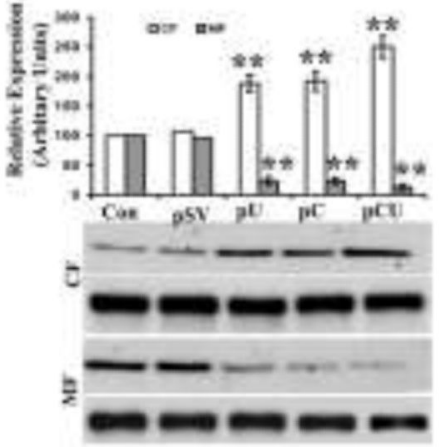

1251
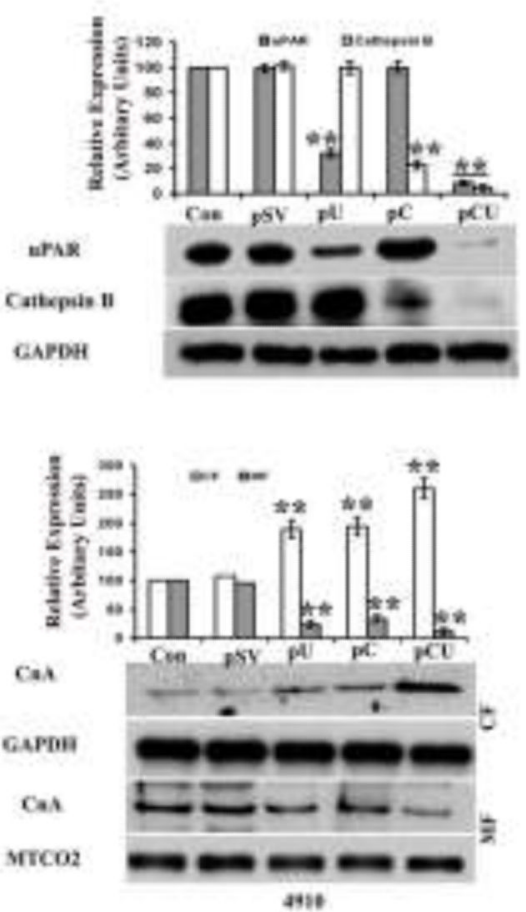

Figure 1. siRNA-mediated downregulation of UPAR and cathepsin B alters localization of calcineurin A

A. Western blot analysis of UPAR and cathepsin B in U251 and 4910 cells after $72 \mathrm{hrs}$ transfection with pSV, pU, pC and pCU. GAPDH was used as a loading control. The graph represents quantitative analysis of UPAR and cathepsin B bands by densitometry. B. Expression of calcineurin A in cytosolic and mitochondrial fractions was determined by Western blot analysis. The graph represents quantitative analysis of calcineurin A bands by densitometry. Total lysates from untreated, pSV-, pU-, pC- and pCU-treated U251 and 4910 cells were fractionated into mitochondrial and cytosolic fractions as per standard protocols and immunoblotted for calcineurin A. MTCO2 was used as a marker for mitochondrial fractions (MF); GAPDH was used for cytosolic fractions $(\mathrm{CF})$. The data represent one of three independent experiments. Values are mean \pm SD of three different experiments $(* * p<0.001)$. 
A)

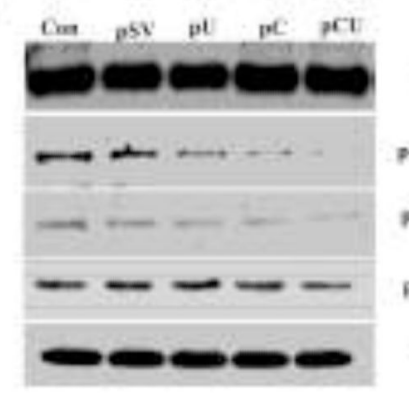

II)

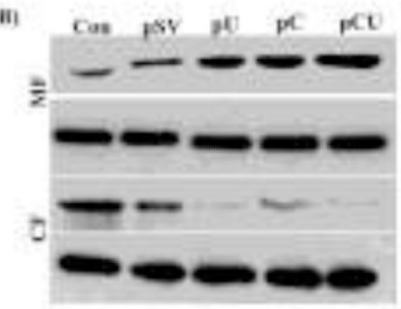

c)

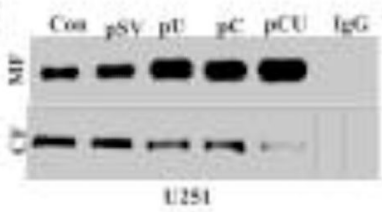

avo

p-8.41)

pacmsiz

p-11.0018155)

c.spn

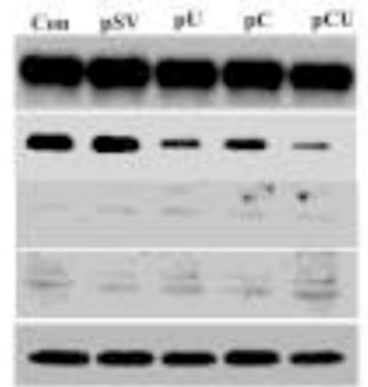

BAล

vrrco?

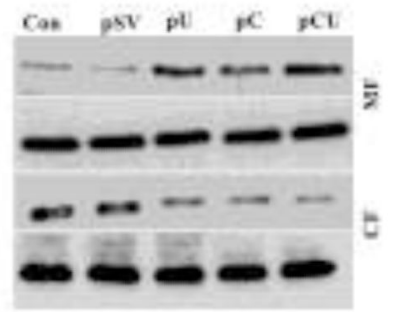

cixpus

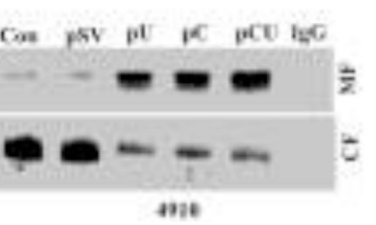

Figure 2. Downregulation of $\mathrm{UPAR}$ and cathepsin $B$ decreases expression of pBAD (S112 and S136), alters localization and interaction of BAD with 14-3-3 and Bcl-xl

A. After transfection, cell lysates were collected from untreated, pSV-, pU-, pC- and pCUtreated cells. Equal amounts of total protein were blotted for expression of BAD and pBAD (S112, S136 and S155). GAPDH was used to verify equal loading. B. Total lysates from untreated and treated U251 and 4910 cells were fractionated into mitochondrial and cytosolic fractions as per standard protocols and immunoblotted for BAD. Expression levels of BAD in cytosolic (CF) and mitochondrial fractions (MF) were determined by western blot analysis. MTCO2 was used as a marker for mitochondrial fractions. GAPDH was used as a loading control for cytosolic fractions. C. Immunoprecipitation analysis of 14-3-3 $\zeta$ and $\mathrm{Bcl}-\mathrm{xl}$ from cytosolic and mitochondrial fractions, respectively after pull down with BAD in untreated and treated cell lysates. IgG was used to check non-specific interaction. 
s)

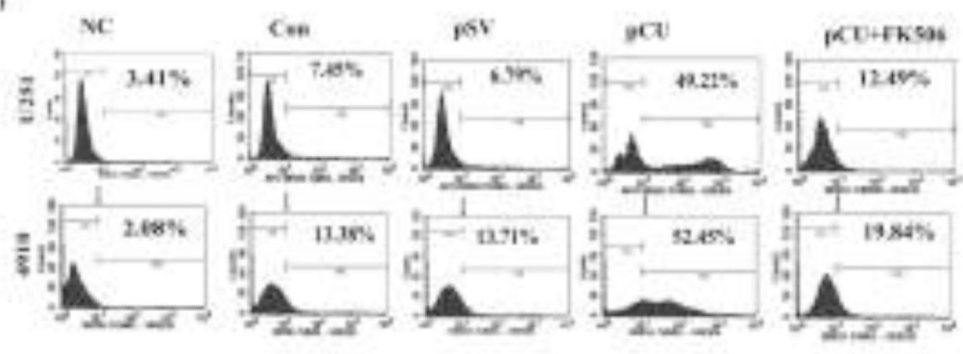

n)

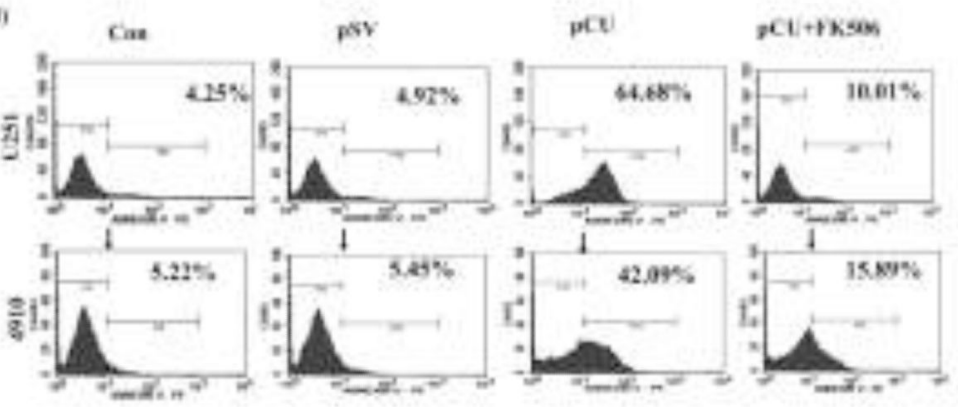

Figure 3. Calcineurin inhibitor (FK506) rescues uPAR and cathepsin B-depleted glioma cells from apoptosis

A. Flow cytometry analysis of pCU-treated U251 and 4910 cells in the presence or absence of FK506 with APO-BrdU-TUNEL. The percentage of TUNEL-positive cells with nuclear DNA strand breaks (emitting green fluorescence) read from the M2 marker, but the cells without fragmented DNA (TUNEL-negative) from the M1 marker. The position of M1 marker was adjusted according to the results of the negative control (without TdT), which allows us to set a region including all TUNEL-negative cells. B. Annexin V-based quantification of pCU-induced apoptosis in the presence or absence of FK506 in U251 and 4910 cells. 
A)
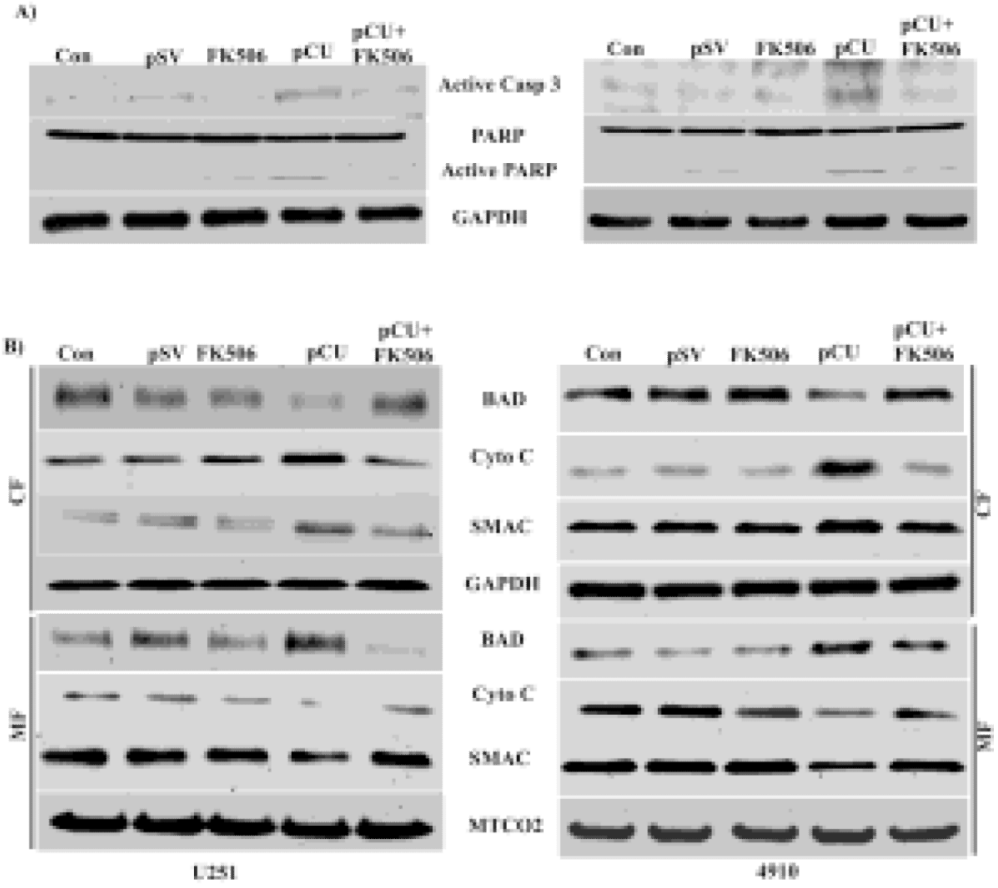

Figure 4. Effect of calcineurin inhibitor on expression of apoptotic molecules in UPAR and cathepsin B-depleted cells

A. U251 and 4910 cells were treated either separately or in combination with pCU and FK506, and immunoblot analysis was performed for expression of active caspase 3 and PARP. B. Expression of BAD, cytochrome $\mathrm{c}$ and SMAC in cytosolic and mitochondrial fractions. After fractionation, cytosolic and mitochondrial fractions were probed for BAD, cytochrome $\mathrm{c}$ and SMAC expression. GAPDH was used as a loading control for cytosolic fractions, and MTO2 was used as a loading control for mitochondrial fractions. 

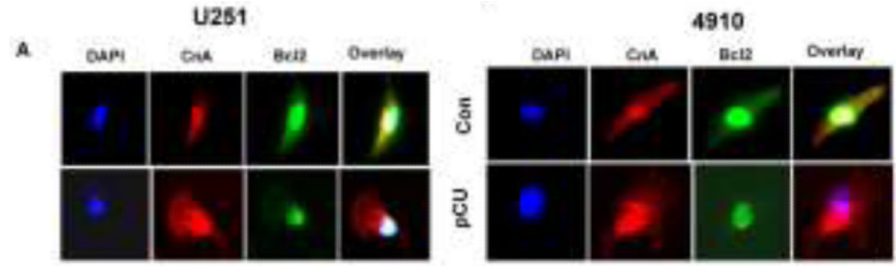

B
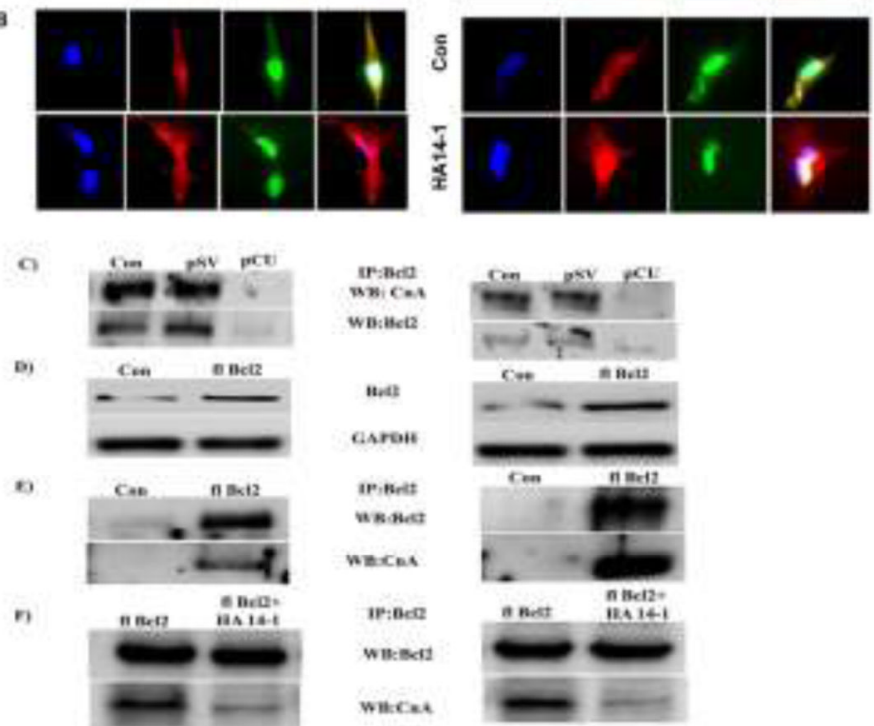

Figure 5. uPAR and cathepsin B knockdown decreases calcineurin A and Bcl-2 interaction A \& B. Immunofluorescence assay was used to detect the co-localization of calcineurin A and Bcl-2 in untreated and pCU-treated or HA 14-1-treated $(20 \mu \mathrm{g} / \mathrm{mL}) \mathrm{U} 251$ and 4910 cells. 72 hrs after treatment, cells were fixed and incubated with calcineurin A and Bcl-2 antibodies followed by incubation with species-specific Alexa Fluor secondary antibody. Total lysates from untreated, pSV-, pCU- or flBcl-2-treated U251 and 4910 cells were immunoprecipitated with Bcl-2 and then immunoblotted for Bcl-2 and calcineurin A. C. Immunoprecipitation analysis of cell lysates collected from UPAR and cathepsin B-depleted cells. D. Expression of Bcl-2 in Bcl-2-overexpressed glioma cells. E. Immunoprecipitation analysis of cell lysates collected from Bcl-2-overexpressed cells. F. Immunoprecipitation analysis of cell lysates collected from Bcl-2-overexpressed cells in the presence or absence of HA 14-1. Cell lysates were immunoprecipitated with Bcl-2 and subjected to western blotting for Bcl-2 and calcineurin A. 

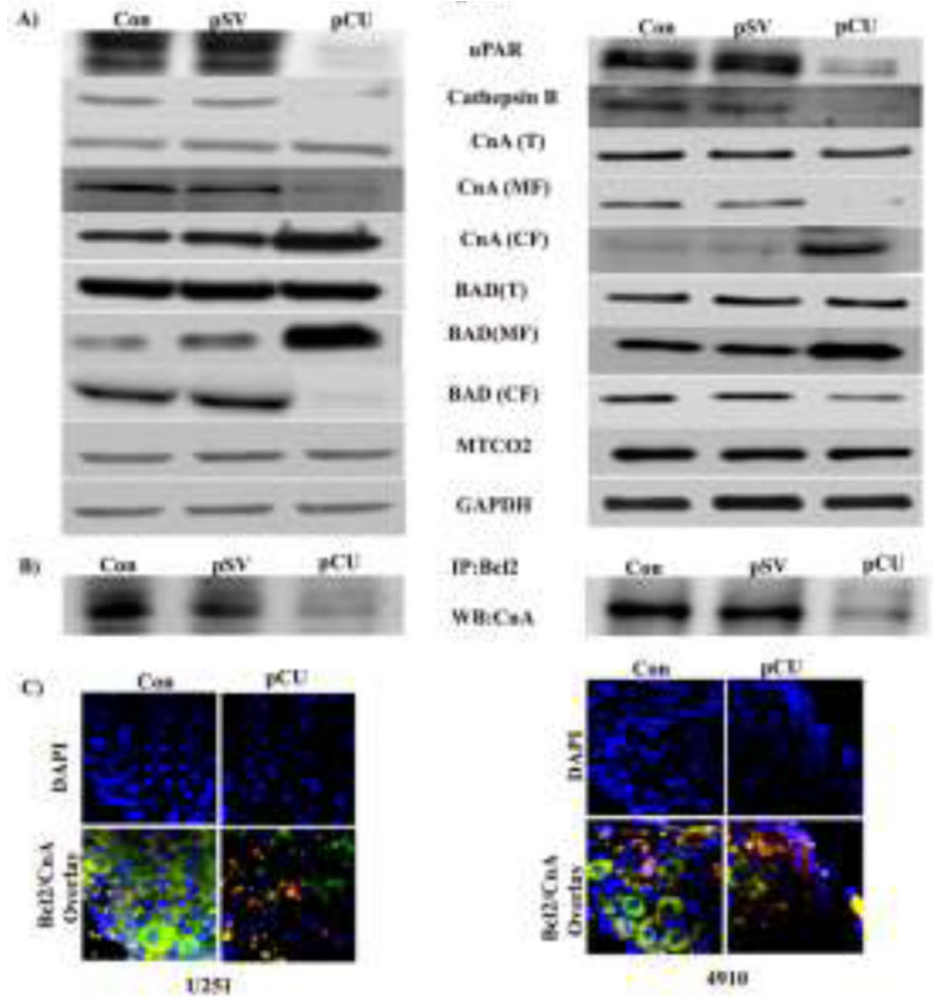

Figure 6. pCU alters localization and interaction of calcineurin A with Bcl-2 in pre-established intracranial tumors

Stereotactic implantation of U251 and $4910\left(1 \times 10^{5}\right)$ tumor cells into nude mice brain was performed. After one week, mock (PBS), pSV or pCU was injected into the brain using an Alzet mini-osmotic pump. Five animals per group were used. 30 days after implantation, the animals were sacrificed, the brains were removed, fixed and paraffin sections were prepared. Fresh brains were used to make tissue lysates. A. Expression of calcineurin A and BAD in total lysate, cytosolic and mitochondrial fractions. Western blot analysis was carried out using specific antibodies. MTCO2 was used as a marker for mitochondrial fractions (MF); GAPDH was used as a marker for cytosolic fractions (CF). B. Immunoprecipitation of calcineurin A, after pull down with Bcl-2, in mitochondrial fractions of brain tissue lysates. C. Co-localization of calcineurin A and Bcl-2 in brain tissue sections. After processing and dehydration as described in Materials and Methods, tissue sections were immunoprobed for calcineurin A (green in U87 and red in 4910) and Bcl-2 (red in U87 and green in 4910) using specific antibodies, followed by species-specific Alexa Fluor-conjugated secondary antibodies. 